\title{
Etude Ethnobotanique, Evaluation De L'activite Antifongique Sur Candida Albicans Et De La Toxicite Sur Des Cellules Hff De Bersama Abyssinica (Fresen.), Une Plante De La Pharmacopee Ivoirienne
}

\author{
Djeneb Camara \\ Kouadio Bene
}

Université Félix Houphouët-Boigny, UFR Biosciences, Laboratoire de

Botanique, Abidjan Côte d'Ivoire

Goueh Gnahoue

Ecole Normal Supérieure (ENS) Abidjan, Laboratoire BiochimieMicrobiologie (Section SVT), Abidjan (Côte d'Ivoire)

N'guessan Bra Yvette Fofie

Laboratoire de Pharmacognosie Botanique et Cryptogamie, UFR Sciences

Pharmaceutiques et Biologiques, Université Felix Houphouët-Boigny,

Abidjan Côte d'Ivoire

Guédé Noël Zirihi

Université Félix Houphouët-Boigny, UFR Biosciences, Laboratoire de

Botanique, Abidjan Côte d'Ivoire

doi: 10.19044/esj.2016.v12n3p171 URL:http://dx.doi.org/10.19044/esj.2016.v12n3p171

\begin{abstract}
To make a contribution to the fight against opportunistic mycoses in sharp increase in AIDS patients, we conducted an ethnobotanical investigation in the Department of Transua (Côte d'Ivoire). This investigation has shown that the plant Bersama abyssinca is the most used in the treatment of skin infection. Our team tested different extracts of leaves of $B$. abyssinica (aqueous extract total (ETA), 70\% ethanol extract (EE70\%) and aqueous extract residual (ERA)) on the in vitro growth of Candida albicans, to determine some inhibitions parameters. These three extracts were active in $C$. albicans, with respective IC $_{50}: 160 \mu \mathrm{g} / \mathrm{ml}$ for ETA, $100 \mu \mathrm{g} / \mathrm{ml}$ for EE70\% and $55 \mu \mathrm{g} / \mathrm{ml}$ for ERA. We found that $B$. abyssinica is not toxic for human cells HFF, on the contrary, it increases the proliferation of dividing cells thereby improving defense mechanisms.
\end{abstract}

Keywords : Bersama abyssinica, antifungal activity, Candida albicans, toxicity, HFF cell 


\section{Résumé}

Dans le but d'apporter notre contribution à la lutte contre les mycoses opportunistes en forte recrudescence chez les malades du SIDA, nous avons réalisé une enquête ethnobotanique dans le Département de Transua en Côte d'Ivoire qui a mis en évidence que Bersama abyssinca est la plante la plus utilisé lors du traitement d’infection cutanée. Notre équipe a testé différents extraits de feuilles de $B$. abyssinica (extrait total aqueux (ETA), extrait éthanolique 70\% (EE70\%) et extrait résiduel aqueux (ERA)) sur la croissance in vitro de Candida albicans afin de déterminer quelques paramètres d'inhibitions. Ces trois extraits ont été actifs sur $C$. albicans, avec des CI50 de $160 \mu \mathrm{g} / \mathrm{ml}$ pour ETA, $100 \mu \mathrm{g} / \mathrm{ml}$ pour EE70\% et $55 \mu \mathrm{g} / \mathrm{ml}$ pour ERA. Nous avons constaté que $B$. abyssinica n'est pas toxique pour les cellules humaines HFF, au contraire, elle augmente la prolifération des cellules en division permettant ainsi d’améliorer les mécanismes de défense.

Mots-clés : Bersama abyssinica, activité antifongique, Candida albicans toxicité, cellules HFF

\section{Introduction}

Les infections opportunistes, surtout celles liées au VIH (SIDA) sont devenues au fil des années un réel problème de santé publique pour les pays en développement. Parmi ces infections opportunistes, les candidoses sont en forte recrudescence, causant ainsi de nombreux décès (Dromer et al., 1996 ). Les candidoses sont des infections dues à des levures; elles sont naturellement présentes sur la peau et dans l'intestin. Au niveau de la peau, elles sont retrouvées dans les plis et sur les fesses, en particulier chez les enfants (Tra Bi et al., 2007). Candida albicans est l'un des principaux responsables. Ce champignon est un pathogène opportuniste, très virulent lorsque la défense immunitaire est faible comme dans le cas du VIH (SIDA).

Les médicaments modernes utilisés dans le traitement des candidoses sont souvent onéreux et inaccessibles à une grande majorité de la population africaine. En Côte d'ivoire, environ $80 \%$ des malades sont suivis et traités par les tradipraticiens (Sanogo et al., 2006). Les plantes médicinales semblent être une bonne alternative aux médicaments modernes. De plus, un des problèmes majeur de ces médicaments modernes est la résistance que l'organisme pathogène met en place (Dupont et al., 1996 ; Li et al., 2015). Il est nécessaire de trouver de nouveau médicaments moins coûteux, accessibles et efficaces. C'est dans cette optique que nous nous sommes intéressés aux plantes médicinales de la pharmacopée ivoirienne, afin d'évaluer sur le plan scientifique leur efficacité sur l'inhibition de $C$. albicans, ainsi que leur toxicité sur des cellules humaines. Pour mieux nous 
orienter dans cette étude mycologique, nous avons réalisé une enquête ethnobotanique afin de recenser les plantes les plus utilisés lors d'un traitement d'infections cutanées.

\section{Le milieu d'étude}

Le Département de Transua, zone de notre investigation est d'une superficie d'environ $1060 \mathrm{Km}^{2}$. Il est limité au Nord par le Département de Bondoukou, à l'Ouest par celui de Tanda, au Sud par le Département de Koun-Fao et à l'Est par le Ghana (pays voisin). Il compte trois souspréfectures à savoir Transua, Assueffry et KouassiaNiaguni. Dans cette étude, nous avons visité dix sites (Figure 1).

Transua a un climat de type tropical humide appartenant au domaine soudanais, caractérisé par deux saisons de pluies et deux saisons sèches. La végétation locale est dominée par la forêt, relativement claire. Le relief est constitué de plaines et de plateaux, traversé par un fleuve la Comoé et ses affluents. Le Département est principalement peuplé par les Brong, mais on y trouve également les Agni et Koulango. Il existe un fort métissage entre ces différents peuples.

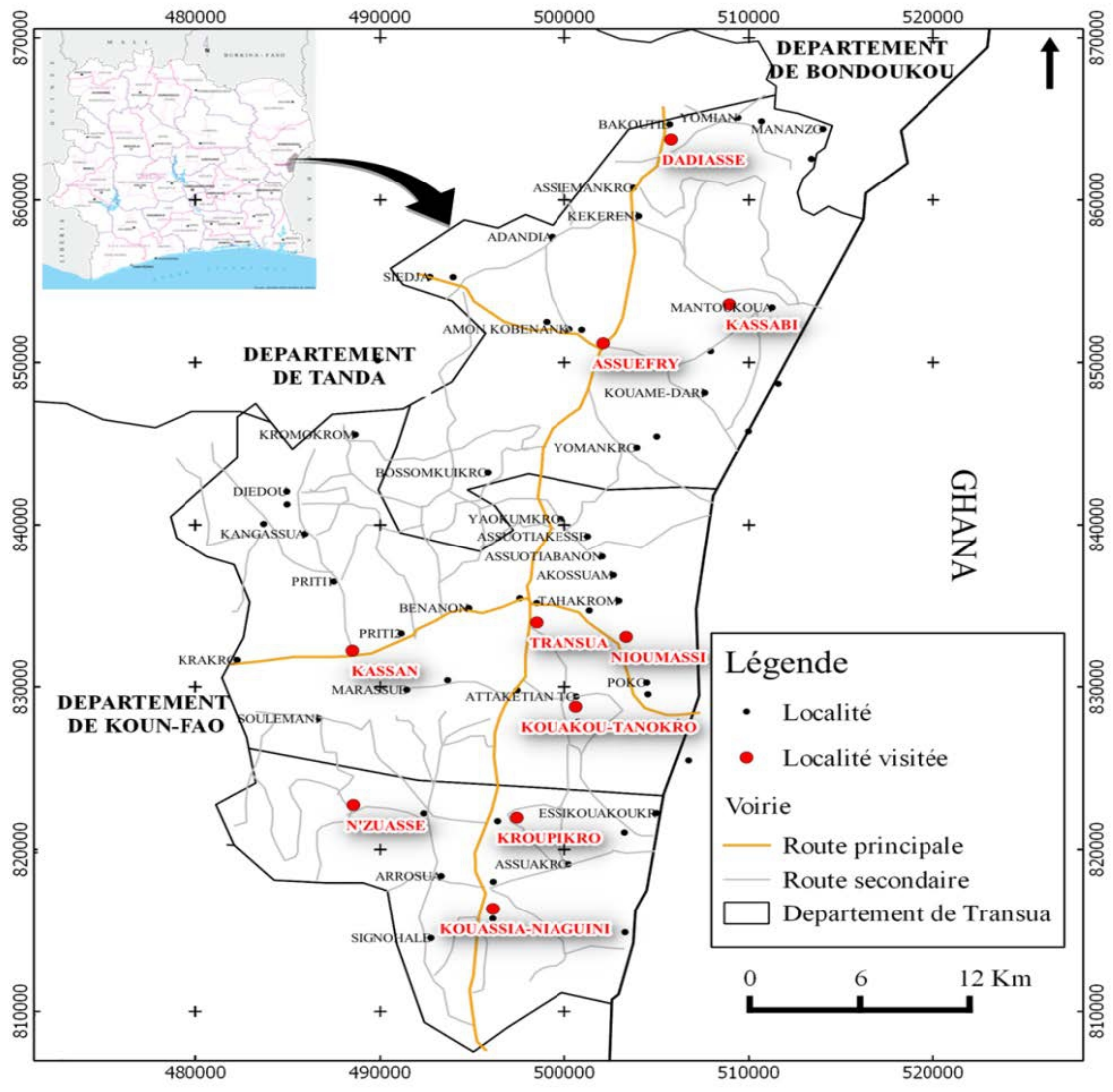

Figure 1 : Situation géographique du Département de Transua 


\section{Matériel}

\section{Matériel végétal : les feuilles de la plante Bersama abyssinica}

Quinze plantes médicinales antimicrobiennes issues du département de Transua ont été inventoriées lors de l'enquête ethnobotanique (Tableau 1). Un échantillon frais de chaque espèce de plante a été prélevé pour la confection d'un herbier en vue de son identification par le Centre National de Floristique (CNF) de l’Université Félix Houphouët-Boigny (UFHB).

Parmi ces plantes, Bersama abyssinica est la plus sollicitée par les populations de cette région. Les feuilles sont les plus couramment utilisées ; elles ont été récoltées pour l'évaluation des activités biologiques in vitro.

\section{Souche fongique : Candida albicans}

L'évaluation de l'activité antifongique a été faite sur un champignon. Il s'agit d'une levure : Candida albicans.

Cette souche provient du Laboratoire de Mycologie de l'Institut Pasteur de Côte d'Ivoire (IPCI). Cette levure a été prélevée sur des malades du SIDA du service des maladies infectieuses du CHU de Treichville. Elle a été isolée sur gélose de Sabouraud contenant du chloramphénicol (Ref : M1067-500G, lot : 0000215703) fourni par HIMEDIA.

\section{Matériel animal : Cellules HFF}

Les cellules HFF (Human Foreskin Fibroblasts) ont été utilisées lors de ce travail. Ce sont des cellules humaines, des fibroblastes issus du derme. Elles ont été modifiées en laboratoire pour être utilisées lors de culture in vitro. Ces cellules ont la particularité de formé un tapis cellulaire après plusieurs jours de culture (96 heures), on dit alors qu'elles sont confluentes, elles arrêtent de se diviser par inhibition de contact. Lorsque ces cellules sont en culture depuis seulement 24 heures, elles sont dans un état de mitose (ou cellules en division).

Ces cellules HFF sont cultivées à $37^{\circ} \mathrm{C}$, sous $5 \%$ de $\mathrm{CO}_{2}$ dans un milieu D10 (Dulbecco Minimum Essential Medium, Gibco, additionné de sérum de veau fœtal $10 \%$; glutamine $1 \%$; pénicilline 50 U.ml-1 et streptomycine $50 \mu \mathrm{g} . \mu \mathrm{l}-1)$.

\section{Méthodes}

\section{Enquête ethnobotanique}

L'enquête ethnobotanique consiste à rencontrer plusieurs acteurs de la pharmacopée traditionnelle qui ont accepté de livrer leurs secrets.

Le guide d'entretien (fiche d'enquête) comportait des questions sur le nom local de l'espèce, les organes ou parties de la plante utilisée, leurs modes de préparation et d'administration des recettes, l'état d'utilisation 
(frais ou sec), le type d’infection traité, etc. Les questions ont été posées dans la langue locale qui est le Brong.

Dans cette étude, la nomenclature des espèces de plantes suit celle de Cronquist (1981).

\section{Préparation des extraits}

Les feuilles de Bersama abyssinica récoltées, ont été rincées à l'eau et séchées à l'abri du soleil. Elles ont ensuite été réduites en poudre fine grâce à un broyeur. Les extraits (total aqueux, éthanolique $70 \%$ et résiduel aqueux) ont été préparés selon la méthode décrite par Zirihi et al. (2003).

\section{Extrait total aqueux}

Cent grammes (100 g) de poudre de drogues sont homogénéisés dans 1 litre d'eau distillée dans un Blender (Mixer) de marque Life’s Superb (LS317) pendant trois fois trois minutes à la température ambiante. L’homogénat obtenu est filtré successivement sur du coton hydrophile puis sur papier WATTMAN (3 mm). A l'aide d'une étuve réglée à $50^{\circ} \mathrm{C}$, le solvant d’extraction est éliminé. L’évaporat sec est récupéré sous forme de poudre et constitue l'extrait total aqueux (ETA).

\section{Fraction éthanol/eau}

Dix grammes (10g) de l'ETA sont dissouts dans $200 \mathrm{ml}$ d'une solution d'éthanol 70\% (70 ml d'éthanol pur 96\% pour $30 \mathrm{ml}$ d'eau distillée) puis homogénéisés dans un Blender. Après décantation dans une ampoule à décanter, le surnageant est recueilli, filtré sur du coton de tout résidu et séché à l'étuve $\left(50^{\circ} \mathrm{C}\right)$. La poudre obtenue constitue l'extrait éthanolique $70 \%$ (EE70\%).

\section{Extrait résiduel aqueux}

La phase inférieure (résiduelle aqueuse) est recueillie et séchée à l'étuve $\left(50^{\circ} \mathrm{C}\right)$. La poudre obtenue constitue l'extrait résiduel aqueux (ERA).

\section{Calcul du rendement}

Le rendement est la quantité d'extrait obtenue à partir de la poudre végétale. Il est exprimé en pourcentage ou est sans unité. En pratique, on a fait le rapport de la masse de l'extrait sur la masse de la poudre végétale qui a servi pour l'extraction qu’on a multiplié par 100. Ceci se traduit par la formule suivante : $r=(m x 100) / M$

( $\mathrm{r}$ : rendement d'extraction ; $\mathrm{m}$ : masse de l'extrait ; $\mathrm{M}$ : masse de la poudre de drogue). 


\section{Activité antifongique}

\section{Préparation des milieux de culture}

L'incorporation des extraits végétaux à la gélose Sabouraud a été faite selon la méthode de la double dilution en tubes penchés (Kra, 2001). Tous les extraits ont été testés séparément. Chaque série comporte 18 tubes à essai dont 2 tubes témoins (un sans extrait végétal, sert de témoin de contrôle de croissancedu germe ; l'autre sans germe et sans extrait sert de témoin de contrôle de stérilité du milieu de culture) et 16 tubes tests (contenant l'extrait végétal).

Pour les seize tubes tests, les concentrations des extraits variaient de 50 à $0,0015 \mathrm{mg} / \mathrm{ml}$ selon une liaison géométrique de raison 1/2.

\section{Stérilisation}

Les 18 tubes de chaque série ont été stérilisés à l'autoclave (PBI STEMATIC III) à $121^{\circ} \mathrm{C}$ pendant 15 minutes et ensuite inclinés avec petit culot à la température de la salle pour permettre leur refroidissement et la solidification de la gélose (Zirihi et al., 2003 et Kporou et al., 2009).

\section{Evaluation de l'activité Antifongique}

L’inoculum est préparé à partir de cultures de germes de 48 heures sur Gélose en pente. Ces germes sont prélevés à l'aide d'une anse de platine, puis homogénéisés dans $10 \mathrm{ml}$ d'eau distillée stérilisée. L'on obtenait ainsi la suspension $10^{0}$ de germes, à partir de laquelle est préparée la suspension $10^{-1}$, par dilution au dixième, en transférant $1 \mathrm{ml}$ de la suspension $10^{0}$ dans $9 \mathrm{ml}$ d'eau distillée pour avoir un volume final de $10 \mathrm{ml}$.

La culture des germes sur les milieux précédemment préparés a été faite par l'ensemencement en stries transversales serrées jusqu'à épuisement de 1000 levures de la souche de C. albicans équivalent à $10 \mu \mathrm{l}$ de la suspension $10^{-1}$ contenant $10^{5}$ germes $/ \mathrm{ml}$ (Holt, 1975).Ces germes sont prélevés à l'aided'une anse de platine.Les cultures ainsi réalisées ont été incubées à la température de la salle pendant 72 heures. Après ce temps d'incubation, les colonies ont été dénombrées par comptage direct grâce à un stylo compteur de colonies de type Geiger. La croissance dans les 16 tubes expérimentaux a été évaluée en pourcentage de survivance, calculé par rapport à $100 \%$ de survivance dans le tube témoin de contrôle de la croissance (Zirihi et al., 2003).

\section{Détermination des paramètres antifongiques (CMI, CMF et $\mathbf{C I}_{50}$ )}

La CMI (Concentration Minimale Inhibitrice) est la plus petite quantité d'extrait à partir de laquelle on n'observe aucune croissance visible à l'œil nu dans le tube à essai. 
Le traitement des données a permis de déterminer graphiquement les différentes valeurs moyennes des $\mathrm{CI}_{50}$ (Concentration pour $50 \%$ d'inhibition) à partir du tracé des courbes de sensibilité de chaque extrait sur C. albicans.

\section{Fongicidie}

Après $72 \mathrm{~h}$ d'incubation, la surface de la gélose contenus dans les tubes tests où l'on n'observe aucune croissance de C. albicans est légèrement prélevée, ensemencée à l'aide d'une anse de platine puis incubée pendant $72 \mathrm{~h}$ sur gélose neutre. Deux cas de figure peuvent se présenter :

- présence de colonies de C. albicans, l'extrait est dit fongistatique.

- absence de colonies de C. albicans, l'extrait est dit fongicide. Cette dernière observation permet de déterminer la CMF (Concentration Minimale Fongicidequi donne 99,99 \% d'inhibition comparativement au tube témoin de contrôle de croissance).

\section{Toxicité sur les cellules HFF}

Cette partie est inspirée de la méthode de Mossman (1983).

Pour mesurer la toxicité de l'extrait éthanolique, les cellules HFF (Human Foreskin Fibroblasts) ont été ensemencées dans des plaques de 96 puits (CellStar) à raison de 3000 à 5000 cellules par puits dans $100 \mu \mathrm{l}$ de milieu D10. Ces cellules sont maintenues en culture pendant 24 heures (cellules en division) ou 96 heures (cellules confluentes). Par la suite elles ont été exposées pendant 24 heures à différentes concentration (0 -1000 $\mu \mathrm{g} / \mathrm{ml})$ en extrait de plante solubilisé dans du tampon PBS. Cela a été fait en triplicate. La viabilité a été déterminée à l'aide du bromure de 3-(4,5diméthylthiazol-2-yl)-2,5-diphenyl tetrazolium (MTT). L'anneau de tétrazolium qu'il contient est réduit en formazan par la succinate déshydrogénase mitochondriale des cellules métaboliquement actives, qui précipite et donne une couleur violette. La quantité du précipité formé est proportionnelle au nombre de cellules vivantes. Dans chaque puits, le MTT est ajouté à une concentration de $500 \mu \mathrm{g} / \mathrm{ml}$ et incubé pendant $3 \mathrm{~h}$ à $37^{\circ} \mathrm{C}$. Les cristaux de formazan sont solubilisés dans du diméthylsulfoxyde (DMSO) 10 $\mathrm{mM}$. La mesure de la densité optique à $544 \mathrm{~nm}$ a été faite à l'aide d'un spectrophotomètre Safir (Tecan) ; cette mesure de l'absorbance permettra de déterminer la quantité relative de cellules vivantes et actives métaboliquement. Les résultats ont été exprimés en pourcentage de viabilité par rapport au contrôle sans extrait de plante.

Taux viabilité $=\left(\mathrm{Abs}_{544 \mathrm{~nm}}\right.$ extrait/ $\mathrm{Abs}_{544 \mathrm{~nm}}$ témoin $) \times 100$.

Dans une première approche, nous avons fait les tests de toxicité sur l'extrait éthanolique (EE70\%) parce qu'il est le plus couramment utilisé en médecine traditionnelle. 


\section{Résultats et discussion Investigations botaniques Analyse floristique}

Les investigations ont permis de différencier quinze espèces de plantes utilisées dans le traitement des maladies de la peau, réparties en quatorze genres et douze familles botaniques (Tableau 1).

Tableau 1 : Données ethnobotaniques sur l'ensemble des plantes recensées

\begin{tabular}{|c|c|c|c|c|}
\hline Taxons & Familles & PU & $\mathrm{TP}$ & MA \\
\hline Ageratum conyzoides L. & Asteraceae & $\begin{array}{l}\text { Rameaux } \\
\text { feuillés }\end{array}$ & Expression & Voie cutanée \\
\hline Bersama abyssinica Fresen. & Meliantaceae & Feuilles & $\begin{array}{l}\text { Décoction } \\
\text { Expression }\end{array}$ & $\begin{array}{l}\text { Voie orale } \\
\text { Voie cutanée }\end{array}$ \\
\hline Biden spilosa L. & Asteraceae & $\begin{array}{l}\text { Rameaux } \\
\text { feuillés }\end{array}$ & Expression & Voie cutanée \\
\hline Calotropis procera W. T. Ait. & Asclepiadaceae & Feuilles & Expression & Voie cutanée \\
\hline Cassia alata L. & Caesalpiniaceae & $\begin{array}{l}\text { Rameaux } \\
\text { feuillés }\end{array}$ & $\begin{array}{l}\text { Décoction } \\
\text { Expression }\end{array}$ & Voie cutanée \\
\hline $\begin{array}{c}\text { Chromolaena odorata (L.) R. M. } \\
\text { King \& H. Robinson }\end{array}$ & Asteraceae & $\begin{array}{l}\text { Rameaux } \\
\text { feuillés }\end{array}$ & Expression & Voie cutanée \\
\hline $\begin{array}{c}\text { Cissus aralioides (Welw, ex Bak.) } \\
\text { Planch. }\end{array}$ & Ampelidaceae & Tige & $\begin{array}{c}\text { Broyage + } \\
\text { amande } \\
\text { douce }\end{array}$ & Voie cutanée \\
\hline Cyathula prostrata (L.) Blume & Amaranthaceae & $\begin{array}{l}\text { Rameaux } \\
\text { feuillés } \\
\text { Infl. }\end{array}$ & $\begin{array}{l}\text { Broyage + } \\
\text { gingembre } \\
\text { Expression }\end{array}$ & Voie cutanée \\
\hline $\begin{array}{c}\text { Kalanchoe crenata (Andrews) } \\
\text { Haw. }\end{array}$ & Crassulaceae & Feuilles & $\begin{array}{c}\text { Expression } \\
+ \text { sel } \\
\text { (Ramollir } \\
\text { au feu) }\end{array}$ & Voie cutanée \\
\hline $\begin{array}{c}\text { Mareya micrantha (Benth.) Müll. } \\
\text { Arg. }\end{array}$ & Euphorbiaceae & $\begin{array}{l}\text { Rameaux } \\
\text { feuillés }\end{array}$ & Décoction & $\begin{array}{l}\text { Voie cutanée } \\
\text { Voie orale }\end{array}$ \\
\hline Mitracarpus scaber Zucc. & Rubiaceae & $\begin{array}{l}\text { Rameaux } \\
\text { feuillés }\end{array}$ & Expression & Voie cutanée \\
\hline Nicotiana tabacum L. & Solanaceae & Feuilles & Expression & Voie cutanée \\
\hline Ocimum gratissimum L. & Lamiaceae & $\begin{array}{l}\text { Rameaux } \\
\text { feuillés }\end{array}$ & $\begin{array}{l}\text { Broyage } \\
\text { (+ piment) }\end{array}$ & Voie cutanée \\
\hline Paullinia pinnata $\mathbf{L}$. & Sapindaceae & $\begin{array}{l}\text { Rameaux } \\
\text { feuillés }\end{array}$ & Décoction & Voie cutanée \\
\hline Solanum erianthum D. Don & Solanaceae & Feuilles & Expression & Voie cutanée \\
\hline
\end{tabular}

PU : Partie Utilisée, TP : Technique de Préparation, MA : Mode d’Administration, Infl. :

Inflorescences. 


\section{Parties de la plante utilisée}

Différentes parties de plante sont utilisées pour les traitements. Ce sont les feuilles (rameaux feuillées) $86,67 \%$, la tige $6,67 \%$ et les inflorescences 6,67\%. Des études menées par d'autres chercheurs confirment que les feuilles sont les partiesles plus utilisées. Les enquêtes ethnobotaniques dirigées par Zirihi (1991) indiquent que les feuilles sont utilisées à 64,49\%. N'Guessan (2008) montre qu'elles sont utilisées à 51,22\%, contre 64\% pour Béné (2014). Le prélèvement intense de ces feuilles ne présente pas de danger pour les plantes.

\section{Technique de préparation}

Trois techniques de préparation sont employées dans le traitement des maladies de la peau: l'expression, la décoction et le broyage. L'expression des organes frais est le plus sollicitée (61,11\%).

\section{Mode d'administration}

Deux voies d'administration (orale et cutanée) sont enregistrées, la voie cutanée 88\% est la plus sollicitée. Diatta et al. (2013) dans leur étude ont montré que la percutanée (46\%) était la voie d'administration la plus employée, cela confirme nos résultats.

Suite à l'enquête ethnobotanique, il ressort que Bersama abyssinica est la plante la plus utilisée.

\section{Etude botanique de Bersama abyssinica}

L'enquête ethnobotanique nous a indiqué que l'une des plantes les plus utilisée lors des affections cutanées est Bersama abyssinica.

\section{Position systématique}

Sous-Genre : Plantae

Classe : Magnoliopsida

Famille : Melianthaceae

Genre : Bersama

Espèce : Bersama abyssinica

Variété : Bersama abyssinica var. paullinioides

\section{Phytogéographie}

Bersama abyssinica fait partie de la famille des Melianthaceae; le genre Bersama comprend huit espèces, toutes en Afrique. Il existe une grande variabilité chez Bersama, cela a entrainé la distinction de nombreuses espèces, sous-espèces et variétés. 
Bersama abyssinica est une plante très répandue en Afrique. Elle est retrouvée en Afrique de l’ouest (excepté au Bénin), dans la corne de l'Afrique et vers le sud du continent (Angola, Zambie, Zimbabwe et Mozambique).

Notre échantillons de $B$. abyssinica a été comparés à celui du Centre National de Floristique : Région de Bondoukou, 07 avril 1966, Aké Assi Nº 8704 ; Lamto, 01 octobre 1982, Fournier Nº1451.

\section{Morphologie externe de Bersama abyssinica}

C'est un arbuste atteignant $6 \mathrm{~m}$ de hauteur, à tronc souvent tortueux. Les feuilles (Figure 2), composées imparipennées, mesurant jusqu’à $60 \mathrm{~cm}$ de longueur, comportent de six à neuf paires de folioles glabrescentes; le rachis est ailé. Les inflorescences, en racèmes solitaires ou par petit nombre, sont axillaires près de l'extrémité des rameaux. Les fleurs, blanches ou jaunâtres, atteignent $2 \mathrm{~cm}$ de longueur. Les fruits, rougeâtres, atteignant $2 \mathrm{~cm}$ de longueur, sont déhiscents à maturité. Les graines sont entourées d'un arille rouge orangé (Aké-Assi 2001).

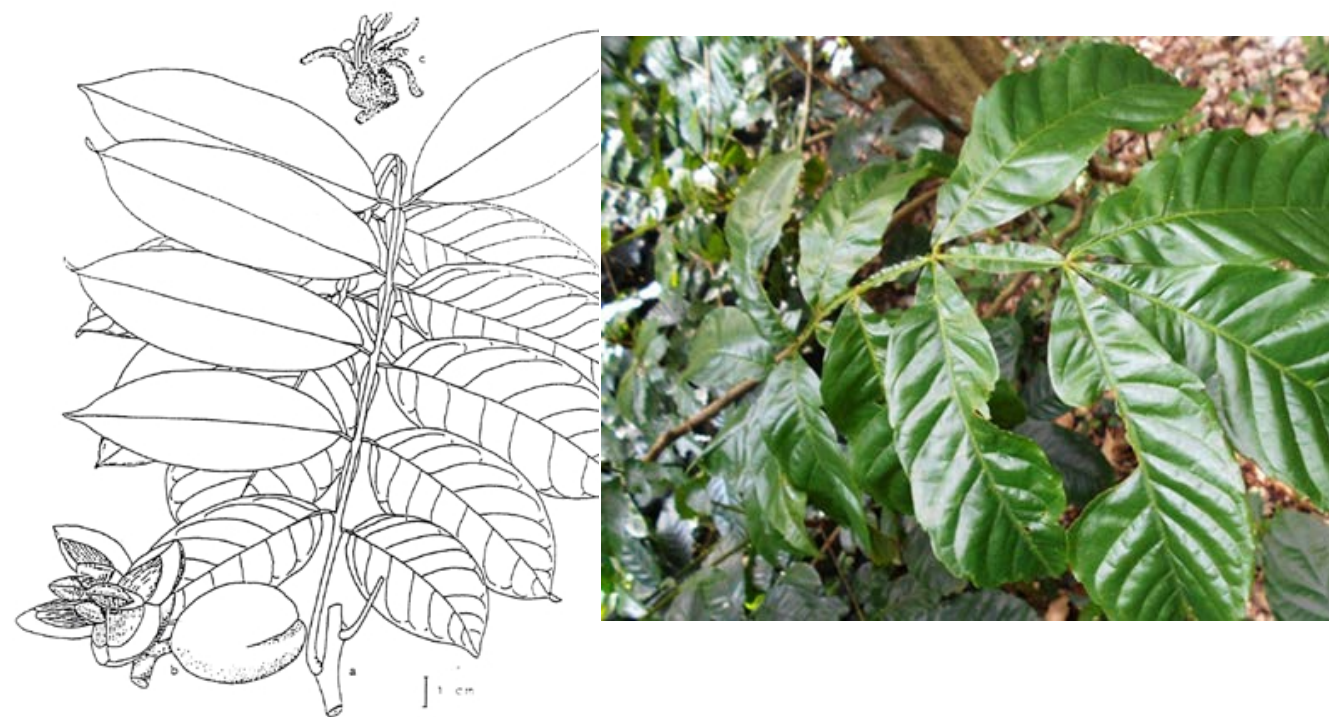
A) a. Rameau feuillé, b. Fruits et
B) Feuille de Bersama abyssinica Fresen. (Photo
c. Fleurs (Planche Zirihi, 2006)
Bene, 2015).

Figure 2 : A et B : Bersama abyssinica Fresen. (Melianthaceae)

\section{Extraction}

\section{Rendement de l'extrait total aqueux (ETA)}

Pour cent (100) grammes de poudre de Bersama abyssinica, on obtient 16,67 grammes d'extraits totaux aqueux soit un rendement de $16,67 \%$.

\section{Rendement de la fraction éthanol/eau}

Les rendements sont consignés dans le tableau 2. 
Tableau 2 : Rendement de la fraction éthanol / eau

\section{Extrait initial de Ba}

Partition

Quantité

Pourcentage

EE70\%

$56 \%$ ETA $(10 \mathrm{~g})$ ERA

$4,20 \mathrm{~g}$

$42 \%$

\section{Effet des extraits de $B$. abyssinica sur $C$. albicans}

Après 72h d'incubation, on observe comparativement au tube témoin, une baisse progressive du nombre de colonies de $C$. albicans au fur et à mesure que les concentrations des extraits ETA, EE70\% et ERA augmentent dans les tubes expérimentaux.

Les valeurs de concentrations inhibitrices obtenues attestent que les extraits ont des activités antifongiques plus ou moins accentuées.

Les courbes des trois extraits présentent une allure décroissante avec des pentes fortes (Figure 3). La pente de ERA est supérieure à celle des EE70\% et de ETA. Les trois courbes coupent l'axe des abscisses à différentes concentrations (respectivement à 98, 195 et 391 et $\mu \mathrm{g} / \mathrm{ml}$ pour ERA, EE70\% et ETA) qui représentent les valeurs des CMI. Cela illustre bien la sensibilité dose dépendante de $C$. albicans aux différents extraits testés.

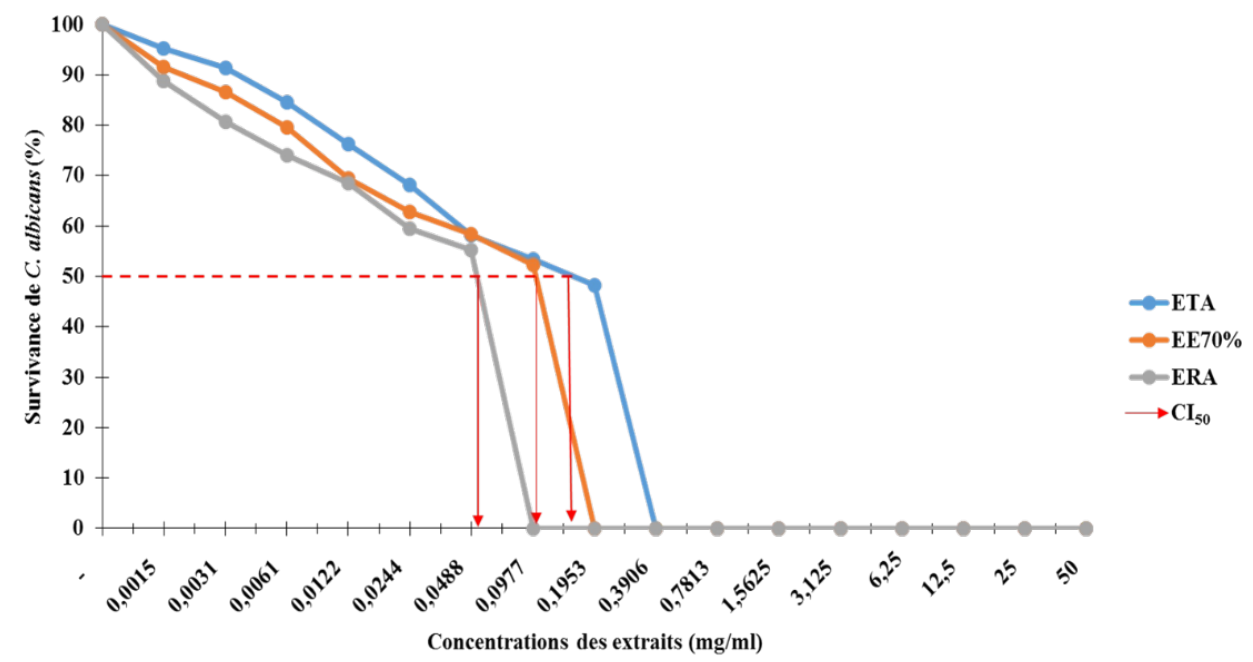

Figure 3 : Sensibilité de Candida albicans aux extraits de B. abyssinica (ETA, EE70\% et ERA)

Les paramètres antifongiques ( $\mathrm{CMI}, \mathrm{CI}_{50}$ et $\mathrm{CMF}$ ) des extraits sont consignés dans le Tableau 3.

Tableau 3 : Valeurs des paramètres antifongiques des extraits ETA, EE70\% et ERA de $B$. abyssinica après 72 heures d'incubation. 


\begin{tabular}{|c|c|c|c|c|}
\hline \multirow{2}{*}{ Extraits de $B a$} & \multicolumn{3}{c|}{ Paramètres antifongiques } & \multicolumn{2}{c|}{ Fongicidie } \\
\hline ETA & $\mathrm{CMI}(\mu \mathrm{g} / \mathrm{ml})$ & $\mathrm{CI}_{50}(\mu \mathrm{g} / \mathrm{ml})$ & CMF $(\mu \mathrm{g} / \mathrm{ml})$ \\
\hline EE70\% & 391 & 160 & SUP50000 & Fongistatique \\
\hline ERA & 195 & 100 & 25000 & Fongicide \\
\hline
\end{tabular}

L'analyse des résultats montre que Candida albicans est sensible aux trois extraits (ETA, EE70\%, ERA) selon une relation dose-réponse.

En comparant les $\mathrm{CI}_{50}(0,055,0,100$ et $0,160 \mathrm{mg} / \mathrm{ml}$ respectivement pour ERA, EE70\% et ETA) et l'allure des courbes, on se rend bien compte que ERA a une meilleure activité que EE70\% et ETA.

Cela pourrait s'expliquer par le fait que la fraction éthanol/eau a permis de séparer les principes actifs et de les concentrer. Cette séparation permet aux principes actifs contenus dans chaque extrait (EE70\% et ERA) de mieux exprimer leur potentiel antifongique. La partition permet donc d'améliorer l'activité antifongique des feuilles de B. abyssinica.

Les travaux de Zekeya et al. (2012) portant sur l'activité antifongique des feuilles de $B$. abyssinca sur $C$. albicans ont montré que l'extrait méthanolique était la plus active avec une CMI de $780 \mu \mathrm{g} / \mathrm{ml}$. En comparant cette valeur à nos résultats, les extraits ETA $(\mathrm{CMI}=391 \mu \mathrm{g} / \mathrm{ml}), \mathrm{EE} 70 \%$ $(\mathrm{CMI}=195 \mu \mathrm{g} / \mathrm{ml})$ et ERA (CMI= $98 \mu \mathrm{g} / \mathrm{ml})$ de B. abyssinca de nos travaux montrent une meilleure activité antifongique par rapport à l'extrait méthanolique de nos prédécesseurs. En établissant le rapport des CMI, on obtient :

$-\mathrm{CMI}_{\text {Méthanolique }} / \mathrm{CMI}_{\mathrm{ETA}}=780 / 391=2$. Ce qui signifie que ETA est deux fois plus actif que l'extrait méthanolique de Zekeya et al. (2012).

$-\mathrm{CMI}_{\text {Méthanolique }} / \mathrm{CMI}_{\mathrm{EE} 70 \%}=780 / 195=4$. Ce qui signifie que EE70\% est quatre fois plus actif que l'extrait méthanolique.

$-\mathrm{CMI}_{\text {Méthanolique }} / \mathrm{CMI}_{\mathrm{ERA}}=780 / 98=8$. Ce qui signifie que ERA est huit fois plus actif que l'extrait méthanolique.

\section{Effet des extraits de B. abyssinica sur les cellules humaines HFF}

L'effet de B. abyssinica sur les cellules humaines est montré dans la figure 4. 


\section{Taux de viabilité des cellules HFF en présence d'un extrait éthanolique de} Bersama Abyssinica

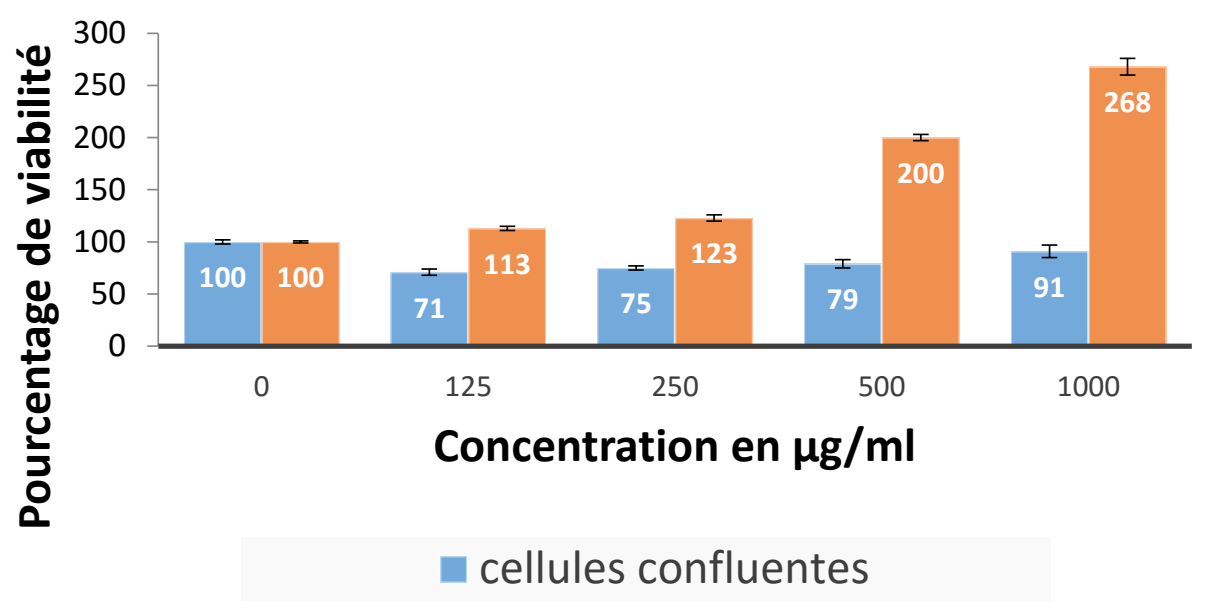

Figure 4 : Toxicité de B. abyssinica sur des cellules humaines HFF

On constate que dans le cas des cellules en division, le taux de viabilité augmente avec la concentration en extrait de plante ; nous avons un effet de l'extrait éthanolique sur la prolifération des cellules. Par contre, dans le cas où les cellules ne se divisent plus, nous n'avons aucun effet dû à la plante. Si cette augmentation de la viabilité était due à une activation de la succinate déshydrogénase utilisé pour réaliser le test MTT, nous aurions le même taux chez les cellules confluentes et les cellules en division. Nous pouvons donc affirmer que dans l'extrait éthanolique il y'a sûrement un facteur de croissance qui augmente la prolifération des cellules aux concentrations étudiées.

Les fibroblastes interviennent dans la défense anti-infectieuse et antivirale par la sécrétion de facteurs chimiostatiques et d'interféron $\beta$. Une augmentation des fibroblastes lors d'une infection de la peau par Candida albicans permettrait à l'organisme de lutter plus efficacement contre le pathogène.

\section{Conclusion}

Notre étude a montré que les extraits ETA, EE70\% et ERA de feuilles de B. abyssinica ont une activité antifongique. Les extraits EE70\% et ERA sont fongicides sur C. albicans et EE70\% de B. abyssinca augmente la prolifération des cellules HFF. Les cellules HFF sont des fibroblastes, des cellules résidentes du derme qui en assurent la cohérence et la souplesse. 
Nous avons donc un double rôle de l'extrait EE70\% de B. abyssinica: destruction de $C$. albicans et augmentation des cellules qui mettent en place le mécanisme de défense. Cette étude justifie bien l’usage de $B$. abyssinica en milieu traditionnel.

\section{References:}

Aké-Assi L., 2001. Flore de la Côte d’Ivoire: catalogue systématique, biogéographie et écologie. Conservatoire et Jardin Botanique, Génève, Switzerland, Boisiera 57; Volume I : 396 p.

Béné K., 2014. Etude ethnobotanique des plantes médicinales utilisées dans le Département de Transua (Côte d'Ivoire). Mémoire de Master d’Ecologie Tropicale (Option Végétale), UFR Biosciences, Université Félix HouphouëtBoigny, Abidjan, Côte d’Ivoire, 50p.

Cunningham A. B., 2002.Appliedethnobotany: People, wild plant use and conservation. People and Conservation Manuel, Earth scan publications Ltd, 300 p.

Diatta C. D., Gueye M. \&Akpo L. E., 2013. Les plantes médicinales utilisées contre les dermatoses dans la pharmacopée Baïnounk de Djibonker, région de Ziguinchor (Sénégal). Journal of Applied Biosciences, 70 : 5599-5607.

Dromer F, Dupont B., 1996. The incrising problem of fungal infections in the immunocompromised host. J. Mycol. Med., 6.

Dupont B, Dromer F, Improvisi L., 1996. The problem of azole resistance in Candida. J. Mycol. Med., 6

Holt J. R., 1975. Laboratory test of antifungal drugs. Journal of Clinical Pathology, 1(18) : 767-774.

Kra A. K. M, 2001. Evalutaion et amélioration par séquençage chromatographique d'une action antifongique de MISCA contre Aspergilus fumigatus. Thèse. Pharma, Bioch. Univ. Abidjan, 126 p.

Kporou K. E., Kra Adou K. M., Ouattara S., Guédé-Guina F., Djaman A. J., 2009. Evaluation de l'activité antifongique de Mitracarpusscaber sur Candida tropicalis. J. sci. pharm. biol., 10(1) : 13-20.

Li L., Naseem S., Sharma S. , Konopka JB., 2015. Flavodoxin-Like Proteins Protect Candida albicans from Oxidative Stress and Promote Virulence. PLoS Pathog. 2015 Sep 1;11(9).

Mossman T., 1983.Rapidcolorimetricassay for cellular growth and survival : application to proliferation and cytotoxicityassay. Journal of immunologicalMethods 65 : 55-63.

N’Guessan K., 2008. Plantes médicinales et pratiques médicales traditionnelles chez les peuples Abbey et Krobou du département d'Agboville (Côte d'Ivoire). Thèse de Doctorat d'Etat ès Sciences, Université de Cocody-Abidjan (Côte d’Ivoire), 235 p. 
ZekeyaN., Shahada F. \&Chacha M., 2012. In vitro Antibacterial and AntifungalActivity of TanzanianBersamaabyssinica. International Journal of Science and Research (IJSR),3: 7.

Zirihi G. N., Kra A. K. M. \&Guédé-Guina F., 2003. Evaluation de l'activité Antifongique de Microglossapyrifolia(LAMARCK) O. KUNTZE (ASTERACEAE) «PYMI » sur la croissance invitrode Candida albicans. Revue de Médecine et de Pharmacopées Africaines ; 17 : 11-18. 